\title{
Source Chaoticity in Relativistic Heavy Ion Collisions at SPS and RHIC
}

\author{
Kenji Morita $^{1 *}$, Shin Muroya ${ }^{2}$, and Hiroki Nakamura ${ }^{1}$ \\ ${ }^{1}$ Department of Physics, Waseda University, Tokyo 169-8555, Japan \\ 2 Matsumoto University, Matsumoto 390-1295, Japan
}

Received on 31 October, 2006; Revised version received on 8 January, 2007; Third version on 26 February, 2007

\begin{abstract}
We investigate degree of coherence of pion sources produced in relativistic heavy ion collisions using multiparticle interferometry. In order to obtain "true" chaoticity, $\lambda^{\text {true }}$ from two-pion correlation functions measured in experiments, we make a correction for long-lived resonance decay contributions. Using this $\lambda^{\text {true }}$ and the weight factor which are obtained from parameter fitted to two- and three-pion correlation function, we calculate a chaotic fraction $\varepsilon$ and the number of coherent sources $\alpha$ for different colliding energies. The result gives constraints on the source and shows an increase of the minimum number of $\alpha$ with multiplicity, although multiplicity independent chaoticity is not excluded.
\end{abstract}

Keywords: Relativistic heavy ion collisions; Pion interferometry

\section{INTRODUCTION}

Relativistic heavy ion collisions provide us an unique opportunity to explore nature of hot and dense nuclear matter on the earth. In the highest energy collisions at the BNL-RHIC, it is expected that the matter created soon after the collision of two heavy nuclei can be the strongly interacting quarkgluon plasma, which gradually cools down and then becomes hadronic matter via phase transitions. To understand the nature of the QCD matter, it is important to know what kind of information experimental observables contain. Pion interferometry has been one of the most important observables because it can give us information on sizes of the source which pions come from, through the HBT effect. The HBT effect is a quantum-mechanical effect due to symmetrization of twoboson wave function and occurs if the source is not completely coherent. The strength of the two-boson momentum intensity correlation takes its maximum value in the case of the perfectly chaotic source. Because the chaoticity can be related to the degree of thermal equilibrium and to composition of the source, analyses of the chaoticity can provide information on a state of hadronic matter which may reflect how hadrons are produced.

Experimentally measured two-pion chaoticity $\lambda=C_{2}(p, p)-1$ does not usually reflect real coherence of the source because of long-lived resonance decay contributions. Three-pion correlation function is more useful for this purpose. The three-pion correlator measured in some experiments is

$$
\begin{aligned}
& r_{3}\left(Q_{3}\right)= \\
& \frac{\left[C_{3}\left(Q_{3}\right)-1\right]-\left[C_{2}\left(Q_{12}\right)-1\right]-\left[C_{2}\left(Q_{23}\right)-1\right]-\left[C_{2}\left(Q_{31}\right)-1\right]}{\sqrt{\left[C_{2}\left(Q_{12}\right)-1\right]\left[C_{2}\left(Q_{23}\right)-1\right]\left[C_{2}\left(Q_{31}\right)-1\right]}},
\end{aligned}
$$

where $Q_{i j}=\sqrt{-\left(p_{i}-p_{j}\right)^{2}}, Q_{3}=\sqrt{Q_{12}^{2}+Q_{23}^{2}+Q_{31}^{2}}$, and $C_{n}$ denotes the $n$-particle correlation function. The weight factor

*Present address: Institute of Physics and Applied Physics, Yonsei University, Seoul 120-749, Korea. Electronic address: morita@phya.yonsei.ac.kr $\omega=r_{3}(0) / 2$ characterizes the degree to how pions sources are chaotic. For a completely chaotic source, $\omega=1$. If the source chaoticity is really a physical quantity, both two- and threepion correlation function should give quantitatively consistent value of the chaoticity. However, experimental results do not seem so, because of the apparent reduction of the $\lambda$ due to long-lived resonances. But it has been shown that we can impose stronger constraints by using both of two- and three-pion correlation data [1].

In this work, we find that such decay contributions can be eliminated by making use of a statistical model [2] and applied it to various experimental data measured in SPS and RHIC experiments [3]. Then, we obtain the weight factor $\omega$ from twoand three-pion correlation function data in these experiments. Using the resonance-corrected $\lambda$ which we call $\lambda^{\text {true }}$ and $\omega$, we calculate a chaotic fraction $\varepsilon$ and the number of coherent sources based on a partially multicoherent source model [4]. From this result, we discuss how the structure of the sources changes from low energy collisions at SPS and higher ones at RHIC [5].

\section{CORRECTION OF $\lambda$ FACTOR FOR LONG-LIVED RESONANCE DECAY}

In the presence of long-lived resonance decay contributions to two-pion correlation function, the two-pion chaoticity for a chaotic source is reduced as

$$
\sqrt{\lambda^{\text {eff }}}=1-\frac{N_{\pi}^{\mathrm{r}}}{N_{\pi}}
$$

where $N_{\pi}$ is the total number of pions and $N_{\pi}^{\mathrm{r}}$ is the number of pions coming from long-lived resonance decay [6]. Hence, if one can estimate the ratio of pions from the decay to the total number, one can obtain apparent reduction factor caused by the decay contribution. Indeed, it is possible with the help of the statistical model which has been known to give a nice description of observed particle number ratio. Under the assumption of the constant chemical freeze-out temperature and chemical potential and boost-invariance along collision axis, the ratio of particle number can be written as the ratio of num- 
ber density, i.e., $N_{i} / N_{j}=n_{i} / n_{j}$, where $n_{i}$ is given by

$$
n_{i}=\frac{g_{i}}{2 \pi^{2}} \int_{0}^{\infty} d p p^{2} f(E, T, \mu)
$$

with $f(E, T, \mu)$ being the equilibrium distribution functions [7]. Using these formulae, we calculate the $\lambda^{\text {eff }}$ for $\mathrm{S}+\mathrm{Pb}$ and $\mathrm{Pb}+\mathrm{Pb}$ collisions at the SPS and $\mathrm{Au}+\mathrm{Au}$ collisions at the RHIC and obtain $\lambda^{\text {true }}$ through a relation $\lambda^{\text {true }}=\lambda^{\exp } / \lambda^{\text {eff }}$ where $\lambda^{\exp }$ is momentum-averaged experimental data [2, 3]. The temperature and baryonic chemical potential are determined by the $\chi^{2}$ fitting to experimental data of particle ratio. See Ref. [3] for details. Here we summarize the result in Table I.

TABLE I: Summary of $\lambda^{\text {true }}$

\begin{tabular}{cccc}
\hline System & $\left(T, \mu_{\mathrm{B}}\right)[\mathrm{MeV}]$ & $\lambda^{\exp }$ & $\lambda^{\text {true }}$ \\
\hline $\mathrm{S}+\mathrm{Pb}(\mathrm{NA44})$ & $(173,196)$ & $0.59(6)$ & $0.94(6)$ \\
$\mathrm{Pb}+\mathrm{Pb}(\mathrm{NA} 44)$ & $(161,223)$ & $0.55(3)$ & $0.98(3)$ \\
$\mathrm{Pb}+\mathrm{Pb}$ (WA98) & $(161,223)$ & $0.58(4)$ & $1.03(4)$ \\
$\mathrm{Au}+\mathrm{Au}(\mathrm{STAR})$ & $(158,36)$ & $0.57(6)$ & $0.93(8)$
\end{tabular}

\section{EXTRACTION OF THE WEIGHT FACTOR}

In order to obtain the weight factor $\omega$, we have to extrapolate experimentally measured $r_{3}\left(Q_{3}\right)$ [Eq. (1)] to $Q_{3}=0$. Using a simple source function in which instantaneous emission and spherically symmetric source are assumed, we construct the two- and the three-pion correlation functions with the formulae

$$
\begin{aligned}
C_{2}\left(p_{1}, p_{2}\right) & =1+\lambda_{\text {inv }} \frac{f_{12}^{2}}{f_{11} f_{22}}, \\
C_{3}\left(p_{1}, p_{2}, p_{3}\right) & =1+v\left(\sum_{(i, j)} \frac{f_{i j}^{2}}{f_{i i} f_{j j}}+2 \frac{f_{12} f_{23} f_{31}}{f_{11} f_{22} f_{33}}\right),
\end{aligned}
$$

where $f_{i j}=1 / \sqrt{\cosh \left(R\left|\mathbf{q}_{i j}\right|\right)}$ is the source function and $R$, $\lambda_{\text {inv }}$ and $v$ are parameters which should be determined by a simultaneous $\chi^{2}$ fit to the two- and the three-pion correlation functions[10]. From a set of these parameters, we can calculate $\omega$ using Eqs. (4),(5) and (1). The results for $\omega$ are shown in Table II.

TABLE II: Results for $\omega$

\begin{tabular}{ccccc}
\hline & $\mathrm{S}+\mathrm{Pb}$ (NA44) & $\mathrm{Pb}+\mathrm{Pb}$ (NA44) & $\mathrm{Pb}+\mathrm{Pb}$ (WA98) & $\mathrm{Au}+\mathrm{Au}$ (STAR) \\
\hline$\omega$ & $0.40 \pm 0.44$ & $1.15 \pm 0.67$ & $0.78 \pm 0.44$ & $0.872 \pm 0.097$ \\
\hline
\end{tabular}

\section{ANALYSIS WITH THE PARTIALLY MULTICOHERENT MODEL}

From considerations in previous sections, we have obtained the two quantities, $\lambda^{\text {true }}$ and $\omega$ as experimental results. Next, we investigate the coherence of the sources using the partially multicoherent model [4]. In this model, there are two characteristic parameters which are related to the $\lambda^{\text {true }}$ and $\omega$ as

$$
\begin{aligned}
\lambda^{\text {true }} & =\frac{\alpha}{\alpha+(1-\varepsilon)^{2}}, \\
\omega & =\frac{2 \alpha^{2}+2 \alpha(1-\varepsilon)^{2}+3(1-\varepsilon)^{3}(1-2 \varepsilon)}{2\left[\alpha^{2}+3 \alpha(1-\varepsilon)^{2}+(1-\varepsilon)^{3}\right]} \sqrt{\frac{\alpha+(1-\varepsilon)^{2}}{\alpha}} .
\end{aligned}
$$

By solving the above equations with respect to $\varepsilon$ and $\alpha$ (this can be analytically), we can obtain allowed regions for $\varepsilon$ and $\alpha$ corresponding to the available range of $\lambda^{\text {true }}$ and $\omega$.

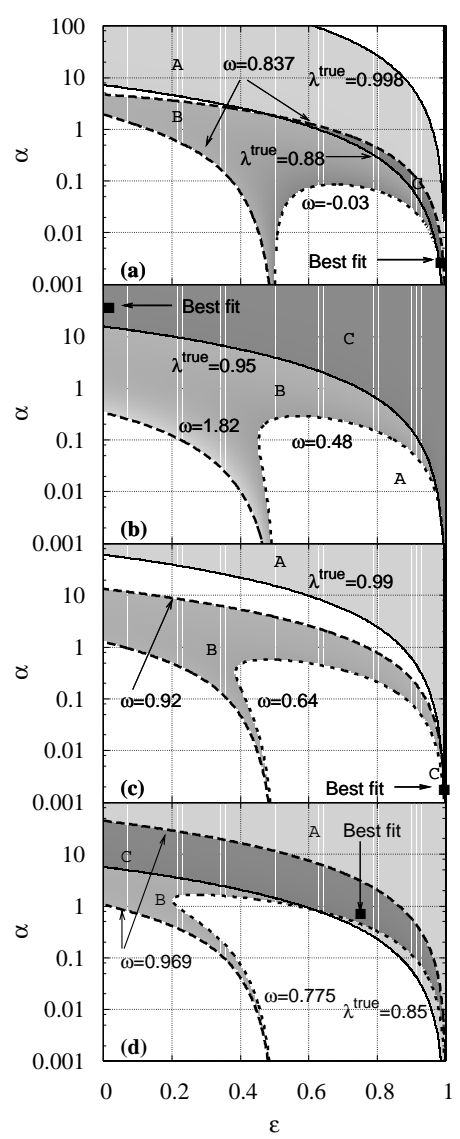

FIG. 1: Allowed region for $\varepsilon$ and $\alpha$. From top to bottom, each figure (a)-(d) shows $\mathrm{S}+\mathrm{Pb}, \mathrm{Pb}+\mathrm{Pb}$ by NA44, $\mathrm{Pb}+\mathrm{Pb}$ by WA98 and $\mathrm{Au}+\mathrm{Au}$ case, respectively.

The result is shown in Fig. 1. In each of figures, the lightest shade area labelled "A" and the second one labelled "B" denote the allowed regions coming from $\lambda^{\text {true }}$ and $\omega$, respectively. The darkest areas labelled " $C$ " are overlap of area "A" and "B", then correspond to the allowed parameter region for $\varepsilon$ and $\alpha$. The best fit points are indicated by the filled box.

From Fig. 1, it seems to be difficult to find systematic change of the allowed regions. This result mainly comes from the fact that $\lambda^{\text {true }}$ 's are close to unity in the $\mathrm{Pb}+\mathrm{Pb}$ data. However, it has been suggested that Coulomb correction to 
the two-pion correlation functions is over-corrected one; the values of $\lambda^{\exp }$ can decrease if we take account of the partial Coulomb correction $[8,9]$. Hence, if appropriate corrections were made, obtained $\lambda^{\text {true }}$ becomes smaller. In order to obtain a rough sketch of a tendency, we also draw the allowed regions for $\lambda_{\mathrm{pc}}^{\text {true }}$ which is 0.8 times smaller than $\lambda^{\text {true }}$. For $\mathrm{S}+\mathrm{Pb}$ data, we multiply the original $\lambda^{\text {true }}$ by factor 0.7 because Gamow (point-like source) correction is made for this data.

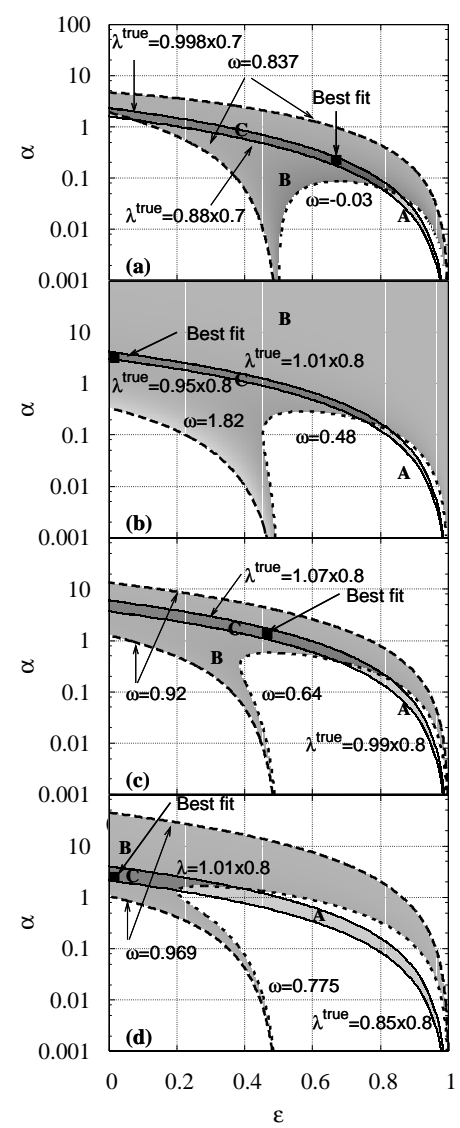

FIG. 2: Allowed region for $\varepsilon$ and $\alpha$, in the case of using $\lambda_{\mathrm{pc}}^{\text {true }}$. From top to bottom, each figure (a)-(d) shows $\mathrm{S}+\mathrm{Pb}, \mathrm{Pb}+\mathrm{Pb}$ by NA44, $\mathrm{Pb}+\mathrm{Pb}$ by WA98 and $\mathrm{Au}+\mathrm{Au}$ case, respectively.

From Fig. 2, we can see that all allowed regions (the darkest shaded areas) are narrow but there seem to exist systematics. The best fit point seems to move upper left (small chaotic frac- tion and large number of coherent sources) side, except for the NA44 $\mathrm{Pb}+\mathrm{Pb}$ result. Most important feature of this result is that the upper limit of $\varepsilon$ and lower limit of $\alpha$ are determined by the lower limit of $\omega$. We plot the maximum and the minimum values of $\alpha$ in Fig. 3. The clear increase of minimum number of the coherent sources can be seen as a function of multiplicity while maximum number of those shows no such clear tendency.

In summary, we have given an analysis of the degree how chaotic the pion sources are in relativistic heavy ion collisions at the SPS and the RHIC. The analysis can be done by using both two-pion correlations and three-pion correlations. We find that the correction for long-lived resonance decay contributions to the two-pion correlation function can be subtracted

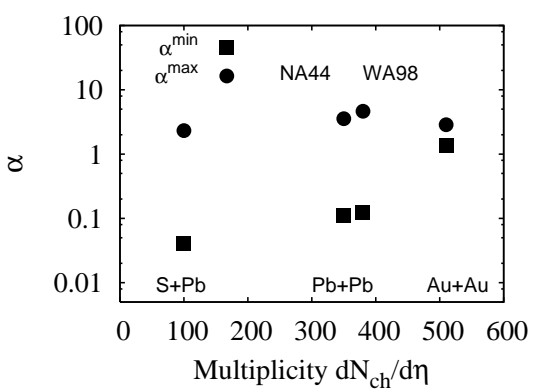

FIG. 3: Maximum and minimum values of $\alpha$ as a function of multiplicity. For $\mathrm{Pb}+\mathrm{Pb}$ data, both plots are slightly shifted along horizontal axis for a clear comparison of the results.

with the help of the statistical model. From a point of view in which multicoherent sources and a background chaotic source are produced, we show that the model gives constraints on the structure of the source. Although the maximum number of the coherent sources does not show a clear multiplicity dependence, the minimum number of coherent source increases as the multiplicity increases.

\section{Acknowledgements}

The authors would like to thank Prof. I. Ohba and Prof. H. Nakazato for their encourgement. K. M's work is supported by a Grant for the 21st Century COE Program at Waseda University from Ministry of Education, Culture, Sports, Science and Technology of Japan and BK21 (Brain Korea 21) program of the Korean Ministry of Education.
[1] H. Nakamura and R. Seki, Phys. Rev. C 66, 027901 (2002).

[2] K. Morita, S. Muroya, and H. Nakamura, Prog. Theor. Phys. 114, 583 (2005).

[3] K. Morita, S. Muroya, and H. Nakamura, Prog. Theor. Phys. 116, 329 (2006). See also references therein.

[4] H. Nakamura and R. Seki, Phys. Rev. C 61, 054905 (2000).

[5] Because of space limitation, here we only show result for the partially multicoherent source model though we also analyzed the data with partial coherent model and multicoherent model in Refs.[2] and [3].
[6] T. Csörgő, B. Lörstad, and J. Zimányi, Z. Phys. C 71, 491 (1996).

[7] J. Cleymans and K. Redlich, Phys. Rev. C 60, 054908 (1999).

[8] D. Adamová et al. (CERES Collaboration), Nucl. Phys. A 714, 124 (2003).

[9] S. S. Adler et al. (PHENIX Collaboration), Phys. Rev. Lett. 93, 152302 (2004).

[10] Eq. (5) is a simple reduction of the correlator part of the fully chaotic case shown in Ref. [4]. See Ref. [2] for discussion. 